\title{
LIGHT-ROASTED GREEN COFFEE EXTRACT IMPROVED ADIPONECTIN, INSULIN RESISTANCE, AND METABOLIC PROFILE OF METABOLIC SYNDROME RAT MODEL
}

\section{MIFETIKA LUKITASARI ${ }^{1}$, DWI ADI NUGROHO ${ }^{2}$, MOHAMMAD SAIFUR ROHMAN ${ }^{3 *}$, NUR IDA PANCA NUGRAHINI ${ }^{4}$, TEGUH WAHYU SARDJONO ${ }^{5}$}

${ }^{1}$ Department of Nursing, Faculty of Medicine, Brawijaya University, Malang, Indonesia. ${ }^{2}$ Department of Basic Medical Science and Biomedicine, Faculty of Medicine, GajahMada University, Indonesia. ${ }^{3}$ Department of Cardiology and Vascular Medicine, Faculty of Medicine, Brawijaya University, Malang, Indonesia. ${ }^{4}$ Department of Agricultural Product Technology, Faculty of Agricultural Technology, Brawijaya University, Malang, Indonesia. ${ }^{5}$ Department of Clinical Parasitology, Faculty of Medicine, Brawijaya University, Malang, Indonesia. Email: mifetika.fk@ub.ac.id

Received: 21 April 2017, Revised and Accepted: 31 May 2017

ABSTRACT

Objective: The objective of this study is to investigate the effect of light-roasted green coffee bean extract (GCE) administration for 7 weeks on the improvement of metabolic profile, adiponectin level, homeostatic model assessment insulin resistance (HOMA-IR) index in metabolic syndrome (MS) rat model.

Methods: Adult male Sprague-Dawley rats were induced by a combination of high sucrose and high-fat diet for 8 weeks and streptozotocin injection in the $2^{\text {nd }}$ week. The MS was confirmed by NCEP-ATP III criteria. They were divided into six weight-matched groups ( $\mathrm{n}=5$ ), normal control, MS, metformin and simvastatin-treated group (DMS), 100 and 200/body weight (bw) GCE (GCE 100 and GCE 200, respectively). The extracts were given through oral gavage daily for 7 weeks. The effect of GCE on body weight, serum glucose, triglyceride, (TG) and high-density lipoprotein (HDL) level was analyzed by colorimetric method. HOMA-IR index and adiponectin were analyzed by enzyme-linked immunosorbent assay methods.

Result: Fasting blood glucose, TG, and systolic blood pressure decreased significantly $(\mathrm{p}<0.05)$ in both GCE groups. Moreover, after 7 weeks, those parameters were significantly lower $(\mathrm{p}<0.05)$ compared to that of MS group. Only GCE 100 group that showed a significant decrease in HDL level. GCE $100 \mathrm{mg} / \mathrm{bw}$ and $200 \mathrm{mg} / \mathrm{bw}$ group showed significantly higher adiponectin level compared to that of MS and DMS group. Furthermore, GCE 100, GCE 200, and DMS group showed a significant lower HOMA-IR index compared to that of MS group.

Conclusion: 7 weeks GCE administration could decrease fasting blood glucose, profile lipid, blood pressure, and improved adiponectin level and HOMA-IR index.

Keywords: Metabolic syndrome, Green coffee bean extract, Adiponectin, Homeostatic model assessment insulin resistance.

(C) 2017 The Authors. Published by Innovare Academic Sciences Pvt Ltd. This is an open access article under the CC BY license (http://creativecommons. org/licenses/by/4. 0/) DOI: http://dx.doi.org/10.22159/ajpcr.2017.v10i9.19329

\section{INTRODUCTION}

Metabolic syndrome (MS) is a complex, multifactor disorder that increases the risk of cardiovascular diseases and diabetes mellitus Type 2. MS prevalence was around $10-84 \%$ based on age, gender, race, ethnicity, and MS criteria [1]. The World Health Organization, International Diabetes Federation, and National Cholesterol of Adult Treatment Panel III have determined specific criteria of MS which included central obesity, high blood pressure, high triglyceride (TG) levels, low high-density lipoprotein (HDL), and high glucose levels [2]. Currently, there is no single treatment for MS, and interest in natural products as potential treatments has increased [3].

Coffee, one of the most widely consumed beverages in the world has attracted a great public interest on its potential health consequences [4]. Coffee has many bioactive components, including caffeine, polyphenols, phenolic acids, and micronutrients. Some epidemiological studies suggested that high phenolic content foods and drinks consumption would prevent coronary artery disease, cancer, and so on $[5,6]$. Chlorogenic acid (CGA) as the most abundant phenolic acid found in green coffee bean may exert antidiabetic, antihyperlipidemia, and antiobesity [7-9] effects. Animal experiments suggested that green coffee bean extract (GCE) rich CGA have beneficial effects on glucose and lipid metabolism disorders in high-fat diet (HFD)-induced mice and rats and can also improve insulin sensitivity in genetically predisposed animals [10]. The effect of GCE could be increased by roasting process.
The previous study suggested that light-roasting process resulted in higher anti-oxidant activity of CGA in GCE [11]. Hence, this study used light roasted coffee beans to make it a novel source of CGAs and eliminating the possible side effects of caffeine.

The effect of coffee consumption on MS improvement was still debated. A study by Cheong et al. revealed that GCE administration for 12 weeks did not attenuate HFD-induced obesity, glucose intolerance, insulin resistance (IR), and systemic oxidative stress in C5BL6 mice [12]. Conversely, Song et al. revealed that decaffeinated GCE attenuated HFDinduced fat accumulation and IR in male C57BL/LN mice [13]. However, there was no prior study that conducted in MS rat model.

Adiponectin, the most abundant circulating adipokine, is a critical regulator of insulin sensitivity [14], tissue inflammation [15], and lipid metabolism [16]. Therefore, adiponectin is considered as an important MS biomarker and therapeutic target $[17,18]$. The effect of GCE administration on adiponectin level and consequently on the improvement of homeostatic model assessment-IR (HOMAIR) index, lipid profile, fasting blood glucose level, and systolic blood pressure (SBP) in MS rat model was still unknown. Thus, this study investigated the effect of light-roasted GCE administration on those therapeutic targets. To the extent of our knowledge, this is the first study revealed the beneficial effect of light-roasted GCE administration on the improvement of adiponectin level, HOMA-IR 
index, lipid profile, fasting blood glucose level, and SBP in MS rat model.

\section{METHODS}

\section{Extraction of green coffee bean}

Coffee was extracted from light roasted robusta green coffee beans (Temanggung, Indonesia). Green coffee bean was sorted to obtain high-quality seed. Green coffee bean weighed $500 \mathrm{~g}$ was dried using a dryer cabinet on the temperature of $50^{\circ} \mathrm{C}$ for $8 \mathrm{~h}$ to obtain simplicia with $8-10 \%$ water content. The simplicia was mashed with a blender and thenmacerated by methanol to produce crude extract. The crude extract was filtered using a filter cloth to separate the liquid phase from the solid phase. The liquid phase was concentrated using rotary evaporator on temperature $\pm 40^{\circ} \mathrm{C}$. The concentrated liquid phase was partitioned using butanol, water, and acetylacetonate. Finally, column chromatography was completed using silica gel as static phase, and the filtered product was evaporated.

\section{Animal care and experimental protocol}

25 Sprague-Dawley rats were purchased from the National Agency of Drug and Food Control, Indonesia. They were housed in standard cages and placed in a room where the temperature was maintained at $25 \pm 1^{\circ} \mathrm{C}$, relative humidity at $50 \pm 1 \%$, and the light at a $12 \mathrm{hrs} \mathrm{light/dark} \mathrm{cycle.}$ During a 1 -week acclimatization period, all rats consumed a normal pellet diet and tap water ad libitum. Afterward, they received high sucrose, HFD, and high sodium diet for 8 weeks and intraperitoneal streptozotocin injection ( $30 \mathrm{mg} /$ body weight [bw]) in the $2^{\text {nd }}$ week. The injection was repeated in the $3^{\text {rd }}$ week with the same dose. The rats with blood glucose (>126 mg/dL), TG (>150 mg/dL), high SBP ( $\geq 140 \mathrm{mmHg}$ ), and reduced HDL levels $(<40 \mathrm{mg} / \mathrm{dL})$ was confirmed as MS rat based on NCEP-ATP III criteria [2]. Furthermore, they were divided into six weight-matched groups $(n=5)$ : The normal control (NC), MS, MS with metformin $200 \mathrm{mg} / \mathrm{bw}$ and simvastatin $10 \mathrm{mg} / \mathrm{kg}$ body weight (DMS), 100 and 200/bw GCE (GCE 100 and GCE 200, respectively). The extract was given through oral gavage daily. Extract dose was given in milliliters based on the body weight measured weekly. The food and water intake was recorded daily. At the end of the experimental period, the animals were anesthetized with ether following a $12 \mathrm{hrs}$ fasting period. Blood samples were drawn from the heart into microcentrifuge tube, and serum samples were obtained by centrifugation at $4000 \times \mathrm{g}$ for 15 minutes at $4^{\circ} \mathrm{C}$. All animal experiments protocol were reviewed and approved by the Ethics Committee of Faculty of Medicine, Brawijaya University.

\section{Physiological measurement}

Daily food intake and fluid intake were measured every day, and body weight was measured every week. The food and fluid intake for each rat were measured by subtracting the provided amount by the remaining amounts in the cage.

\section{Biochemical analysis}

The serum concentrations of TG, HDL-cholesterol, and fasting glucose were measured enzymatically using commercial kits (Biolabo, France). Serum adiponectin and insulin levels were analyzed using enzymelinked immunosorbent assay method (Elabscience, China). The HOMAIR was used to calculate an index from the product of the fasting concentrations of plasma glucose $(\mathrm{mg} / \mathrm{dl})$ and insulin $(\mu \mathrm{u} / \mathrm{L})$ divided by 14.1. Lower HOMA-IR values indicate greater insulin sensitivity and higher HOMA-IR values indicate IR.

\section{Blood pressure measurements}

Blood pressure was measured using the tail-cuff method with sphygmomanometer technique at the baseline and at the end of the experiment. Three readings were taken consecutively, and the average was then calculated and taken as a final reading for SBP.

\section{Statistical analysis}

All data were analyzed with Statistical Package for Social Sciences (SPSS, version 22) and were presented as mean values with their standard deviation and subjected to one-way ANOVA, independent t-test, and pair t-test with significant $\mathrm{p}<0.05$.

RESULT

Effect of GCE on changes in the body weight, food, and water intake The body weight, food, and water intake of experimental rats were elevated during 7 weeks. However, at the end of study, no significant difference was observed in those variables among all groups as presented in Tables 1 and 2, respectively. Body weight increased significantly in 200 CGE group during 7-week intervention. Moreover, a significant food intake increase was observed in DMS group. Furthermore, a significant water intake increase was observed in all experimental groups during 7 weeks.

\section{Effects of GCE on blood biochemistry}

Effect of GCE on fasting blood glucose, TG, and HDL cholesterol on the experimental animals were presented in Tables 3 and 4 . The level of fasting blood glucose TG and HDL was not statistically different among all groups at the baseline. Furthermore, after intervention for 7 weeks, a significant difference on fasting blood glucose, and TG level was observed in all interventional groups $(\mathrm{p}<0.05)$ compared to that of the baseline. At the end of study, a significant difference on fasting blood glucose and TG level was observed in GCE 100, GCE 200, and DMS group compared to that of MS group. The lowest blood fasting glucose level was observed in DMS and GCE 100 groups respectively. Moreover, the lowest TG level was observed DMS and GCE 200 group, respectively. No statistically different observed on HDL level among all interventional group.

\section{Effect green coffee on blood pressure}

GCE 100 and GCE 200 group showed a significant SBP decrease after intervention for seven weeks compared to that of the baseline. At the end of study, a significant lower SBP was observed in GCE 100 and GCE 200 group compared to that of MS group (Table 4).

\section{Effects of GCE on plasma adiponectin}

Effect of GCE on plasma adiponectin level was presented in Fig. 1. GCE 100 and GCE 200 group showed significantly higher adiponectin level compared to that of MS and DMS group. Moreover, administration of GCE at a dose of $100 \mathrm{mg} / \mathrm{kg}$ bw resulted in a slightly higher adiponectin level compared to that of $200 \mathrm{mg} / \mathrm{kg}$ bw.

\section{Effects of GCE on HOMA-IR index}

Effect of GCE on HOMA-IR index was presented in Fig. 2. GCE 100, GCE 200, and DMS group showed significantly lower HOMA-IR index compared to that of MS group. Moreover, GCE 100 group showed lower HOMA-IR index compared to that of GCE 200 group although it was not achieve any significant statistical difference. At the end of study, a significant different of HOMA-IR index was observed in GCE 100 and GCE 200 group compared to that of MS group $(\mathrm{p}<0.05)$.

\section{DISCUSSION}

A recent study was conducted in rat model that met the criteria of MS (hyperglicemia, elevated TG level, decreased HDL level, and

Table 1: Effect of GCE on changes in the body weight of normal and MS rats

\begin{tabular}{lll}
\hline Experimental groups & \multicolumn{2}{l}{ Body weight $\mathbf{( g )}$} \\
\cline { 2 - 3 } & Initial & Final \\
\hline NC & $297.80 \pm 10.66$ & $411 \pm 9.3$ \\
MS & $336.4 \pm 14.7$ & $386.4 \pm 57.17$ \\
GCE 100 & $298.4 \pm 39.87$ & $311 \pm 59.74$ \\
GCE 200 & $301 \pm 44.32$ & $351.6 \pm 74^{\#}$ \\
DMS & $318 \pm 30.05$ & $318.8 \pm 44.07$ \\
\hline
\end{tabular}

Values are mean $\pm S D, n=5$. "Significant between baseline and after

7 week $(p<0.05)$. NC: Normal control, MS: Metabolic syndrome, GCE: Green coffee extract 
Table 2: Effect of GCE on changes in food intake and fluid intake of normal and metabolic syndrome rats

\begin{tabular}{|c|c|c|c|c|}
\hline \multirow[t]{2}{*}{ Experimental groups } & \multicolumn{2}{|c|}{ Food intake (g) } & \multicolumn{2}{|c|}{ Fluid intake (ml) } \\
\hline & Initial & Final & Initial & Final \\
\hline $\mathrm{NC}$ & $21.82 \pm 1.48$ & $26.85 \pm 1.9$ & $33.86 \pm 0.77$ & $20.31 \pm 1.1$ \\
\hline GCE 100 & $18.2 \pm 1.48$ & $45.2 \pm 4.88$ & $66.4 \pm 4.92^{\#}$ & $17.8 \pm 3.27$ \\
\hline GCE 200 & $19.38 \pm 2.58$ & $42.97 \pm 13.46$ & $64.15 \pm 8.98^{\#}$ & $20.03 \pm 5.12$ \\
\hline DMS & $18.87 \pm 2.54$ & $41.81 \pm 9.02$ & $59.48 \pm 7.42^{\#}$ & $26.58 \pm 2.39$ \\
\hline
\end{tabular}

Values are mean \pm SD, $n=5$. "Significant between baseline and after 7 week ( $<<0.05)$. NC: Normal control, MS: Metabolic syndrome, GCE: Green coffee extract,

SD: Standard deviation

Table 3: Effect of GCE on changes in fasting blood glucose and TG of normal and metabolic syndrome rats

\begin{tabular}{|c|c|c|c|c|}
\hline \multirow[t]{2}{*}{ Experimental groups } & \multicolumn{2}{|c|}{ Fasting blood glucose (mg/dl) } & \multicolumn{2}{|l|}{ TG (mg/dl) } \\
\hline & Initial & Final & Initial & Final \\
\hline $\mathrm{NC}$ & $92.20 \pm 16.84$ & $95.2 \pm 11.45$ & $79.40 \pm 7.02$ & $100 \pm 24.13$ \\
\hline GCE 100 & $260 \pm 26.16$ & $186.20 \pm 11.10^{* \#}$ & $281.40 \pm 142.76$ & $153.40 \pm 15.79^{* \#}$ \\
\hline GCE 200 & $247.80 \pm 29.21$ & $201.20 \pm 11.3^{* \#}$ & $262.40 \pm 62.91$ & $146.8 \pm 14.94^{* \#}$ \\
\hline DMS & $248 \pm 28.67$ & $178.40 \pm 59.94^{* \#}$ & $296.40 \pm 30.51$ & $158.2 \pm 42.31^{* \#}$ \\
\hline
\end{tabular}

Values are mean \pm SD, $n=5$. \#Significant between baseline and after 7 week ( $p<0.05)$. *Significant compared to that of MS group ( $<<0.05)$. NC: Normal control,

MS: Metabolic syndrome, TG: Triglyceride, SD: Standard deviation, GCE: Green coffee extract

Table 4: Effect of GCE on changes in HDL-cholesterol and SBP of normal and MS rats

\begin{tabular}{|c|c|c|c|c|}
\hline \multirow[t]{2}{*}{ Experimental groups } & \multicolumn{2}{|c|}{ HDL-cholesterol (mg/dl) } & \multicolumn{2}{|c|}{ SBP (mm/Hg) } \\
\hline & Initial & Final & Initial & Final \\
\hline $\mathrm{NC}$ & $44.40 \pm 3.13$ & $44.80 \pm 3.03$ & $125.2 \pm 5.16$ & $126.8 \pm 6.41$ \\
\hline GCE 100 & $27.60 \pm 8.17$ & $40.40 \pm 5.77^{\#}$ & $152.6 \pm 3.84$ & $145.4 \pm 3.43^{* \#}$ \\
\hline GCE 200 & $34.60 \pm 5.45$ & $39.20 \pm 2.86$ & $152.4 \pm 7.40$ & $147.0 \pm 8.03^{* \#}$ \\
\hline DMS & $29 \pm 8.09$ & $43 \pm 7.07$ & $158.4 \pm 1.94$ & $157.2 \pm 1.78$ \\
\hline
\end{tabular}

Values are mean \pm SD, $n=5$. "Significant between baseline and after 7 weeks $(\mathrm{p}<0.05)$. ${ }^{*}$ Significant compared to that of MS group (p<0.05). NC: Normal control,

MS: Metabolic syndrome, SBP: Systolic blood pressure, SD: Standard deviation, HDL: High-density lipoprotein, GCE: Green coffee extract

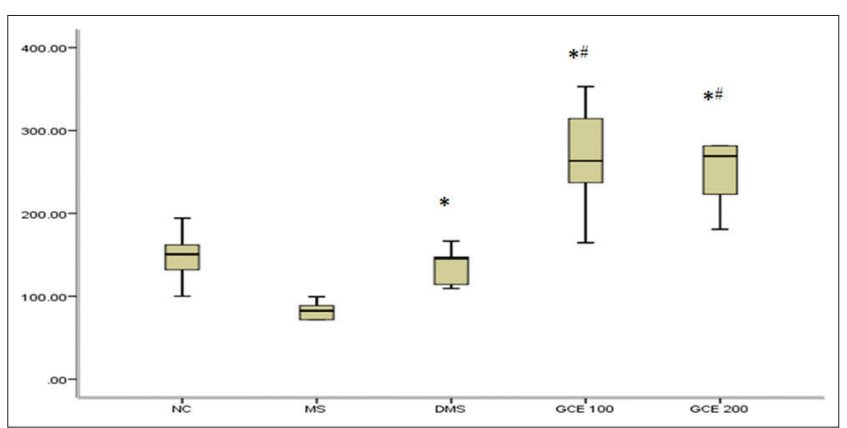

Fig. 1: Effect of green coffee bean on adiponectin level blood serum in normal control, metabolic syndrome (MS), DMS, green coffee extract (GCE) 100, and GCE 200 rats. Values are mean \pm standard deviation, $n=5 .{ }^{*} p<0.01$, compared to the MS group and ${ }^{*} p<0.05$ compared to the DMS group. NC: Normal control; MS: Metabolic syndrome induces; DMS: Metabolic syndrome treated with metformin and simvastatin; GCE 100: Metabolic syndrome with green coffee extract $100 \mathrm{mg} / \mathrm{kg}$ body weight (bw); GCE 200: Metabolic syndrome with green coffee extract $200 \mathrm{mg} / \mathrm{kg} \mathrm{bw}$

hypertension) as presented in baseline characteristics in Table 2. This study revealed that GCE 100 and GCE 200 administration improved MS. It was confirmed by high adiponectin level, low HOMA-IR index, significant reduction of blood glucose, TG, and SBP in GCE 100 and GCE 200 group.

Adiponectin is a key molecule in the pathogenesis of MS [19]. In the present study, GCE improved metabolic profile of MS through adiponectin increase $[20,21]$. This study demonstrated a significantly higher adiponectin level in GCE 100 and GCE 200 compared to that of MS group. The adiponectin level also closed to that of NC group. This study was comparable with the previous study by Cho et al. that revealed a significantly higher adiponectin level after 8 weeks administration of $0.02 \%$ CGA in diet compared to that of obese mice group [20]. Moreover, a study by Ong et al. suggested that 2 weeks treatment of CGA from green coffee bean improved serum adiponectin level in Lepr ${ }^{\mathrm{db} / \mathrm{db}}$ mice compared to that of diabetic mice [10]. To the extent of our knowledge, this study was the first study that proved the benefit of CGE in MS rat.

Adiponectin level increasing effect by GCE was mediated by CGA induce transcriptional activity of peroxisome proliferator-activated receptor gamma (PPAR- $\gamma$ ). The previous study by Wu et al. showed that 200 and $400 \mathrm{mg}$ of CGA for 12 weeks increased transcriptional activity of PPAR- $\gamma$ and consequently adiponectin level in $\mathrm{ApoE}^{-/}$mice [22]. It was revealed that PPAR- $\gamma$ affected adiponectin by controlling the expression of the adiponectin genes in adipose tissue. Moreover, adiponectin increasing effect was also shown in DMS group compared to that of MS group. Nevertheless, the effect of metformin and simvastatin only restoring the adiponectin level as high as NC group's level. This result was consistent with the previous study that suggested metformin effect on restoring the adiponectin level as high as normal rat [23]. Thus, GCE administration demonstrated a better adiponectin increasing effect compared to that of $200 \mathrm{mg} / \mathrm{kg}$ bw metformin and $10 \mathrm{mg} / \mathrm{kg}$ bw simvastatin administration

This study revealed that adiponectin level of GCE 100 was higher than that of GCE 200 although it was not achieve any statistical significance. In contrast, Choi et al. suggested that higher GCE dose resulted in higher adiponectin level [21]. The differences of substances composition 


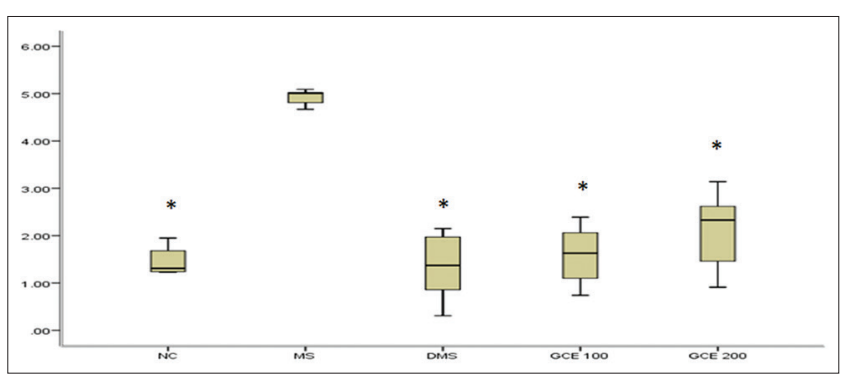

Fig. 2: Effect of green coffee bean on homeostatic model assessment insulin resistance index in normal control (NC), metabolic syndrome (MS), DMS, green coffee extract (GCE) 100, and GCE 200 rats. Values are mean \pm standard deviation, $n=5$. ${ }^{*} \mathbf{p}<0.01$ compared to the MS group. NC: Normal control; MS: Metabolic syndrome induces; DMS: Metabolic syndrome treated with metformin and simvastatin; GCE 100: Metabolic syndrome with green coffee extract $100 \mathrm{mg} / \mathrm{kg}$. body weight (bw); GCE 200 Metabolic syndrome with green coffee extract $200 \mathrm{mg} / \mathrm{kg} . \mathrm{bw}$

in the extract might contribute to this result. The extract used in our study was not purified, some caffeine and other polyphenols might contain in our extract on the other hand, Choi et al. used GCE contained 50\% of caffeoylquinic acid (CQA) [21]. The chronic effect of higher GCE dose administration on homocysteine level might explain lower adiponectin level in higher GCE dose. The previous studies demonstrated that chronic administration of high-dose green coffee raised blood homocysteine level [24]. The mechanisms by which CGA raise plasma homocysteine are not clear. The previous study suggested that caffeine content in GCE might contribute significanly to the high level of homocystein. The previous study revealed that pure caffeine consumption increased homocysteine concentrations in healthy subjects $[25,26]$. However, caffeine from paper-filtered coffee only contributed to $25-50 \%$ of homocysteine increase compared to that of a similar amount of caffeine [25].

High homocystein level has been proved as a suppresor of adiponectin level. The previous study suggested that liver might affect adipocyte physiology through adipokines expression regulation through the secretion of metabolic molecules such as homocysteine [27]. The previous study clearly demonstrated that multiple pathways were involved in this mechanism. Homocysteine induced both inhibition of adiponectin gene expression in primary adipocytes and reduced circulating adiponectin levels in an animal model of mild hyperhomocysteinemia [27]. It revealed that elevated homocysteine in adipose tissue may play a causal role in suppressing adiponectin production.

Adiponectin also known as insulin sensitizer agent. The present study suggested that higher adiponectin negatively correlated with HOMA-IR index [14]. In our study, we assumed that higher adiponectin level led to lower HOMA-IR index. GCE 100 group who had higher adiponectin level showed lower HOMA-IR index compared to that of GCE 200 and DMS group. Although there was no significant, HOMA-IR difference among GCE 100, GCE 200, and DMS. The obtained HOMA-IR index by GCE 100 closed to that of NC group. These findings are consistent with previous reports that adiponectin functions as an insulin sensitizer by decreasing hepatic glucose output and thereby contributing to the regulation of whole-body glucose homeostasis [28]. Consistent with this, restoration of adiponectin levels in vivo by CGA from green coffee showed improvement of glycemia, insulin sensitivity, and fatty acid metabolism [29]. Moreover, it could have enhanced the activation of adenosine monophosphate-activated protein kinase (AMPK) through calmodulin-dependent protein kinase in muscle and liver [10] and upregulated AdipoR2 expression that resulted in insulin sensitivity modulation by stimulation of glucose utilization and fatty acid oxidation through phosphorylation [30]. These mechanisms were considered as beneficial effect of GCE in metabolic alterations.
Recently, this study showed that $100 \mathrm{mg} / \mathrm{kg}$ bw GCE was the effective dose in treating hyperglycemia, dyslipidemia in rat model of MS for 7 weeks. The extent of GCE $100 \mathrm{mg} / \mathrm{kg}$ bw decreased fasting blood glucose, and TG level was also shown in GCE $200 \mathrm{mg} / \mathrm{kg}$ bw. However, the fasting blood glucose and TG level were slightly lower than GCE $100 \mathrm{mg} / \mathrm{kg}$ bw. In contrast, the previous study by Shimoda showed that an oral administration of GCE (200 and $400 \mathrm{mg} / \mathrm{kg}$ day) for 13 days resulted in a tendency ofserum TG level reduction in mice [31]. Moreover, Choi et al. reported that an oral administration of GCE 100 and GCE 200 in C57BL mice reduced serum glucose, TG level after 6 weeks, and significantly difference compare to that HFD group [21]. On the other hand, in significant serum HDL increase in GCE 100 and GCE 200 group was observed in this study. Antihyperglycemia and antidyslipidemia of GCE might be mediated by CGA activity. CGA affected AMPK signaling pathway [32], upregulated expression of adiponectin [10], upregulated adiponectin receptor gene [30], and increased transcriptional factor of PPAR- $\alpha$ [20] and PPAR- $\gamma[21]$ which could regulate glucose and lipid metabolism.

A recent study revealed no body weight lowering effect after GCE administration for 7 weeks using both doses. This result was consistent with a study by Panchal et al. that showed no body weight lowering effect of $5 \%$ aqueous coffee extract oral administration for 8 weeks in MS rat model induced by HFD and high carbohydrate [33]. In contrast, other study revealed that GCE administration resulted in a tendency of visceral fat and body weight reduction. Song et al. suggested that the administration of $0.3 \%$ decaffeinated GCE for 11 weeks in HFD-fed mice resulted in body weight reduction [13]. Shimoda et al. also suggested that the administration of $0.5 \%$ and $1 \%$ GCE reduced visceral fat content and body weight.

The present study shows that GCE 100 and $200 \mathrm{mg} / \mathrm{bw}$ reduced blood pressure from $152.6 \pm 3.84$ to $145.4 \pm 3.43$ and $152.4 \pm 7.40$ to $147.0 \pm 8.03$, respectively. However, blood pressure increased was observed in MS group and DMS group. The blood pressure lowering effect of GCE was first reported in 2002 in spontaneus hypertensive rat (SHR) rat [34]. The previous study by Panchal et al. demonstrated that oral administration of $5 \%$ aqueous coffee extract after 8 weeks. SBP were reduced by $10-15 \mathrm{mmHg}$ from baseline (measured by tail cuff), and the effect persisted through the end of the experiment [33]. As mentioned previously, GCE are rich in CGAs, with the principal form being 5-CQA. The presence of 5-CQA was hypothesized to promote SBP reduction. Both acute and chronic administration of 5-CQA showed almost identical results on SBP as seen in GCE administration on MS animal model diet induced. Furthermore, several mechanisms of SBP reduction by CGA and it metabolites such as, inhibition of nicotinamide adenine dinucleotide phosphate oxidase expression and activity, reduced free radical production, directly scavenging free radicals, stimulation of NO production by the endothelial-dependent pathway, and inhibition of angiotensin-converting enzyme in the plasma [35]. Hypotension effect of GCE could be influenced by roasting condition which that increase antioxidant activity [36].

Roasting effect on coffee can increase the antioxidant effect of polyphenols contained in coffee. Castillo et al. analyzed various roasted degree of coffee beansby gel filtration chromatography, ultravioletvisible spectrophotometry, capillary electrophoresis, and the 2,2 '-azinobis(3-ethylbenzthiazoline-6-sulfonic acid) assay. It revealed that maximum antioxidant activity was observed inlight to medium roasted coffee; unroasted and dark coffee had significantly lower antioxidant activity compared to that of light to medium roasted coffee [11]. We assumed that this antioxidant activity increase contributed to antidiabetic, antidyslipidemia, and antihypertensive effect of GCE. However, we did not measure the antioxidant activity and composition our extract. Furthermore, as limitation of our study, we did not analyze another metabolic inflammation marker such as leptin, high-sensitivity C-reactive protein, AMPK activity, PPAR $\alpha$, and PPAR $\gamma$ gene expression; therefore, we cannot reveal GCE pathway on MS improvement. Further study regarding these issues is warranted. 


\section{CONCLUSION}

This study revealed the beneficial effect of light-roasted GCE administration on the improvement of adiponectin level, HOMA-IR index, lipid profile, fasting blood glucose level, and SBP in MS rat model.

\section{ACKNOWLEDGMENT}

Authors are thankful to KEMENRISTEK DIKTI and University of Brawijaya for providing the funding support to the present investigation.

\section{REFFERENCES}

1. Wild SH, Byrne CD. The epidemiology of the metabolic syndrome and its association with diabetes, cardiovascular disease and other conditions. Metab Syndr 2011;1:1-18.

2. Grundy SM, Brewer HB Jr., Cleeman JI, Smith SC Jr., Lenfant C; American Heart Association; National Heart, Lung, et al. Definition of metabolic syndrome: Report of the National Heart, Lung, and Blood Institute/American Heart Association conference on scientific issues related to definition. Circulation 2004;109(3):433-8.

3. Markolf Hanefeld US, Schaper F. Treatments for the metabolic syndrome. Metab Syndr 2011;327-345.

4. Islam MT, Alencar M, Mata A, Paz M, Matos LA, Sousa JM, et al. Coffee: A health fuel-blot popular drinking. Int J Pharm Pharm Sci 2016;8(1):1-7.

5. Wang Y, Ho CT. Polyphenolic chemistry of tea and coffee: A century of progress. J Agric Food Chem 2009;57(18):8109-14

6. Yukawa GS, Mune M, Otani H, Tone Y, Liang XM, Iwahashi H, et al. Effects of coffee consumption on oxidative susceptibility of lowdensity lipoproteins and serum lipid levels in humans. Biochemistry (Mosc) 2004;69(1):70-4

7. Greenberg JA, Boozer CN, Geliebter A. Coffee, diabetes, and weight control. Am J Clin Nutr 2006;84(4):682-93.

8. van Dam RM. Coffee and type 2 diabetes: From beans to beta-cells. Nutr Metab Cardiovasc Dis 2006;16(1):69-77.

9. Bollapragada MK, Shantaram M, Sunil Kumar R. Obesity: Development, epidemiology, factors affecting, quantity, health hazards, management and natural treatment - A review. Int J Pharm Pharm Sci 2017;9(2):12.

10. Ong KW, Hsu A, Tan BK. Anti-diabetic and anti-lipidemic effects of chlorogenic acid are mediated by AMPK activation. Biochem Pharmacol 2013;85(9):1341-51.

11. Del Castillo MD, Ames JM, Gordon MH. Effect of roasting on the antioxidant activity of coffee brews. J Agric Food Chem 2002;50(13):3698-703

12. Li Kwok Cheong JD, Croft KD, Henry PD, Matthews V, Hodgson JM, Ward NC. Green coffee polyphenols do not attenuate features of the metabolic syndrome and improve endothelial function in mice fed a high fat diet. Arch Biochem Biophys 2014;559:46-52.

13. Song SJ, Choi S, Park T. Decaffeinated green coffee bean extract attenuates diet-induced obesity and insulin resistance in mice. Evid Based Complement Alternat Med 2014;2014:718379.

14. Tschritter O, Fritsche A, Thamer C, Haap M, Shirkavand F, Rahe S, et al. Plasma adiponectin concentrations predict insulin sensitivity of both glucose and lipid metabolism. Diabetes 2003;52(2):239-43.

15. Aldhahi W, Hamdy O. Adipokines, inflammation, and the endothelium in diabetes. Curr Diab Rep 2003;3(4):293-8.

16. Lafontan M, Viguerie N. Role of adipokines in the control of energy metabolism: Focus on adiponectin. Curr Opin Pharmacol 2006;6(6):580-5.

17. Matsuzawa Y, Funahashi T, Kihara S, Shimomura I. Adiponectin and metabolic syndrome. Arterioscler Thromb Vasc Biol 2004;24(1):29-33.

18. Ryo M, Nakamura T, Kihara S, Kumada M, Shibazaki S, Takahashi M, et al. Adiponectin as a biomarker of the metabolic syndrome. Circ J 2004;68(11):975-81.

19. Okamoto Y, Kihara S, Funahashi T, Matsuzawa Y, Libby P. Adiponectin: A key adipocytokine in metabolic syndrome. Clin Sci (Lond) 2006;110(3):267-78.

20. Cho AS, Jeon SM, Kim MJ, Yeo J, Seo KI, Choi MS, et al. Chlorogenic acid exhibits anti-obesity property and improves lipid metabolism in high-fat diet-induced-obese mice. Food Chem Toxicol 2010;48(3):937-43

21. Choi BK, Park SB, Lee DR, Lee HJ, Jin YY, Yang SH, et al. Green coffee bean extract improves obesity by decreasing body fat in high-fat diet-induced obese mice. Asian Pac J Trop Med 2016;9(7):635-43.

22. Wu C, Luan H, Zhang X, Wang S, Zhang X, Sun X, et al. Chlorogenic acid protects against atherosclerosis in ApoE-/- mice and promotes cholesterol efflux from RAW264.7 macrophages. PLoS One 2014;9(9):e95452.

23. Adamia N, Virsaladze D, Charkviani N, Skhirtladze M, Khutsishvili M. Effect of metformin therapy on plasma adiponectin and leptin levels in obese and insulin resistant postmenopausal females with type 2 diabetes. Georgian Med News 2007;145(4):52-5.

24. Olthof MR, Hollman PC, Zock PL, Katan MB. Consumption of high doses of chlorogenic acid, present in coffee, or of black tea increases plasma total homocysteine concentrations in humans. Am J Clin Nutr 2001;73(3):532-8

25. Verhoef P, Pasman WJ, Van Vliet T, Urgert R, Katan MB. Contribution of caffeine to the homocysteine-raising effect of coffee: A randomized controlled trial in humans. Am J Clin Nutr 2002;76(6):1244-8.

26. Urgert R, Van Vliet T, Zock PL, Katan MB. Heavy coffee consumption and plasma homocysteine: A randomized controlled trial in healthy volunteers. Am J Clin Nutr 2000;72(5):1107-10.

27. Song Z, Zhou Z, Deaciuc I, Chen T, McClain CJ. Inhibition of adiponectin production by homocysteine: A potential mechanism for alcoholic liver disease. Hepatology 2008;47(3):867-79.

28. Kadowaki T, Yamauchi T, Kubota N, Hara K, Ueki K, Tobe K. Adiponectin and adiponectin receptors in insulin resistance, diabetes, and the metabolic syndrome. J Clin Invest 2006;116(7):1784-92.

29. Chirumbolo S. In vivo anti-diabetic potential of chlorogenic acid as a consequence of synergism with other phenolic compounds? Br J Nutr 2015;113(3):546-7.

30. Jin S, Chang C, Zhang L, Liu Y, Huang X, Chen Z. Chlorogenic acid improves late diabetes through adiponectin receptor signaling pathways in $\mathrm{db} / \mathrm{db}$ mice. PLoS One 2015;10(4):e0120842.

31. Shimoda H, Seki E, Aitani M. Inhibitory effect of green coffee bean extract on fat accumulation and body weight gain in mice. BMC Complement Altern Med 2006;6(1):9.

32. Ong KW, Hsu A, Tan BK. Chlorogenic acid stimulates glucose transport in skeletal muscle via AMPK activation: A contributor to the beneficial effects of coffee on diabetes. PLoS One 2012;7(3):e32718.

33. Panchal SK, Poudyal H, Waanders J, Brown L. Coffee extract attenuates changes in cardiovascular and hepatic structure and function without decreasing obesity in high-carbohydrate, high-fat diet-fed male rats. J Nutr 2012;142(4):690-7.

34. Suzuki A, Kagawa D, Ochiai R, Tokimitsu I, Saito I. Green coffee bean extract and its metabolites have a hypotensive effect in spontaneously hypertensive rats. Hypertens Res 2002;25(1):99-107.

35. Zhao Y, Wang J, Ballevre O, Luo H, Zhang W. Antihypertensive effects and mechanisms of chlorogenic acids. Hypertens Res 2012;35(4):370-4.

36. Hügel HM, Jackson N, May B, Zhang AL, Xue CC. Polyphenol protection and treatment of hypertension. Phytomedicine 2016;23(2):220-31. 